\title{
Multistimulation Group Therapy in Alzheimer's Disease Promotes Changes in Brain Functioning
}

Neurorehabilitation and

Neural Repair

2015, Vol. 29(I) 13-24

(C) The Author(s) 2014

Reprints and permissions: sagepub.com/journalsPermissions.nav DOI: 10.1 I77//5459683/4532833 nnr.sagepub.com (SAGE

\author{
Francesca Baglio, MD', Ludovica Griffanti, PhD ${ }^{1,2}$, Francesca Lea Saibene', \\ Cristian Ricci, PhD',3, Margherita Alberoni, MD', Raffaella Critelli', \\ Fabiana Villanelli', Raffaella Fioravanti', Federica Mantovani', \\ Alessandra D'amico', Monia Cabinio, PhD ${ }^{1,4}$, Maria Giulia Preti, PhD ${ }^{1,2}$, \\ Raffaello Nemni, MD ${ }^{1,4}$, and Elisabetta Farina, MD ${ }^{\prime}$
}

\begin{abstract}
Background. The growing social emergency represented by Alzheimer's disease (AD) and the lack of medical treatments able to modify the disease course have kindled the interest in nonpharmacological therapies. Objective. We introduced a novel nonpharmacological approach for people with AD (PWA) named Multidimensional Stimulation group Therapy (MST) to improve PWA condition in different disease domains: cognition, behavior, and motor functioning. Methods. Enrolling 60 PWA in a mild to moderate stage of the disease, we evaluated the efficacy of MST with a randomizedcontrolled study. Neuropsychological and neurobehavioral measures and functional magnetic resonance imaging ( $\mathrm{fMRI}$ ) data were considered as outcome measures. Results. The following significant intervention-related changes were observed: reduction in Neuropsychiatric Inventory scale score, improvement in language and memory subscales of Alzheimer's Disease Assessment Scale-Cognitive subscale, and increased fMRI activations in temporal brain areas, right insular cortex, and thalamus. Conclusions. Cognitive-behavioral and $\mathrm{MMRI}$ results support the notion that MST has significant effects in improving PWA cognitive-behavioral status by restoring neural functioning.
\end{abstract}

\section{Keywords}

Alzheimer's disease, rehabilitation, occupational therapy, recreation therapy, cognitive therapy, magnetic resonance imaging, MRI, functional MRI, language

\section{Introduction}

Alzheimer's disease (AD) is a neurodegenerative disorder affecting multiple clinical domains involving cognitive functioning, behavioral aspects, and functional-physical skills. ${ }^{1}$ In the past 15 years, the growing social emergency represented by $\mathrm{AD}$ and the lack of medical treatments able to modify the disease course have kindled the interest in nonpharmacological therapies. This term, often used to describe the array of approaches and techniques proposed in the literature, spans several domains (cognitive-oriented therapies, physical medicine, etc). Another term used to indicate these kinds of approaches is psychosocial therapies, but this word seems to exclude the possibility that these therapies can modify brain function or structure. Hence, some authors have proposed modified versions such as biopsycosocial or psychobiosocial therapies. ${ }^{2,3}$ Functional magnetic resonance imaging (fMRI), due to its capacity to monitor neural circuits reorganization, has been recently introduced to evaluate the efficacy of these nonpharmacological treatments (see Cramer et $\mathrm{al}^{4}$ for a review), also including treatments for amnestic mild cognitive impairment (MCI $)^{5}$ and $\mathrm{AD} .^{6}$

Cognitive stimulation (CS) is probably the most widespread and well-studied technique in this field, but it is still a matter of debate whether this therapy is able to slow down the disease course. ${ }^{7}$ The most defined form is cognitive stimulation therapy (CST), which incorporates the positive aspects of reality orientation therapy but avoids putting the person with dementia in stressful situations and implements

\footnotetext{
'IRCCS, Don Gnocchi Foundation, Milan, Italy

${ }^{2}$ Politecnico di Milano, Milan, Italy

${ }^{3}$ University of Regensburg, Regensburg, Germany

${ }^{4}$ Università degli Studi di Milano, Milan, Italy

\section{Corresponding Author:}

Francesca Baglio, Magnetic Resonance Laboratory and Neurorehabilitation Unit, Fondazione Don Carlo Gnocchi ONLUS, IRCCS, S. Maria Nascente, Via Capecelatro 66, Milan 20I48, Italy. Email: fbaglio@dongnocchi.it
} 
stimulation in a respectful and person-centered manner. ${ }^{8}$ In 2003, Spector and colleagues ${ }^{9}$ showed that this approach was at least as effective as cholinesterase inhibitors to reduce cognitive decline. On the basis of this work, the 2006 NICE guidelines concluded that this intervention should be routinely proposed to people with mild to moderate dementia. ${ }^{10}$ Recently, a maintenance protocol with CST was developed with a very precise procedure that involved not only professionals but also people with dementia and their caregivers. ${ }^{11}$ It seems that CST was able to change some aspects of cognition, especially those related to language and memory, more than others. ${ }^{12,13}$ In a Cochrane review on $\mathrm{CS},{ }^{14}$ the authors found a consistent benefit on cognitive function that persisted at follow-up 1 to 3 months after the end of the treatment. In secondary analyses with smaller total sample sizes, improvements were also observed on self-reported quality of life and well-being.

In the past years, further nonpharmacological therapy studies have allowed for firmer conclusions to be drawn on their efficacy. Occupational and recreational therapy, for example, can reduce psychobehavioral disturbances of people with dementia, increase their participation, improve their quality of life, reduce negative communication, extend the caregiver sense of competence, and reduce his/her burden. ${ }^{15,16}$ Moreover, exercise has been shown to improve the physical health and the well-being of people with dementia ${ }^{17}$ and appeared to be beneficial in reducing behavioral and psychological symptoms of dementia (BPSD). ${ }^{18}$ Interestingly, aerobic exercise training in adults seems to promote an improvement in spatial memory and to reverse age-related loss in volume by 1 to 2 years. ${ }^{19}$ In Belgium and the Netherlands, psychomotor therapy has been well integrated into mental health care since $1965 .^{20}$

Based on this growth of evidence, some research groups have therefore decided to propose multidimensional protocols of treatment for people with AD (PWA), based on the hypothesis that this type of approach would be the most suitable to improve their condition in different disease domains: cognition, behavior, and motor functioning. This kind of therapy would be also the most appropriate for the unique profile of each person with $\mathrm{AD}$, even when performed in small groups. ${ }^{21,22}$ In the past 13 years, our group has developed a multidimensional approach-Multidimensional Stimulation Therapy (MST). Our previous findings ${ }^{23-25}$ support the notion that this group activity program, based on cognitive stimulation, recreational-occupational activities, and physical-psychomotor exercises, can lead to an improvement in behavioral aspects for PWA.

In this article, we describe a randomized single-blind trial aimed to clarify the efficacy of an MST program in outpatients affected by AD in mild to moderate stages of the disease. We also included fMRI as a surrogate marker of treatment efficacy.

\section{Methods}

\section{Participants}

Sixty participants were consecutively recruited from the Don Gnocchi Foundation Memory Clinic.

Outpatients eligibility criteria were the following: $(a)$ diagnosis of probable AD according to NINCDS-ADRDA criteria $^{1} ;(b)$ evidence of $\mathrm{AD}$ pathophysiological processes detected with structural MRI as a biomarker of neural injury ${ }^{1}$; (c) mild to moderate stage of AD (Mini-Mental State Examination (MMSE) score of 15-24 [see Magni et $\mathrm{al}^{26}$ ] and Clinical Dementia Rating scale score of 1-2 [see Hughes et $\left.\mathrm{al}^{27} \mathrm{~J}\right) ;(d)$ age range 65 to 85 years and school attendance range 5 to 17 years; $(e)$ right handedness as assessed by the Edinburgh inventory. ${ }^{28}$ PWA were excluded if they had $(a)$ severe aphasia (Token test score $<20$ [see Spinnler and Tognoni $\left.{ }^{29}\right]$ ) or severe auditory and/or visual loss; $(b)$ overt severe behavioral disturbances that could hinder the MST session; and (c) recent ( 3 months before the MST) introduction or dose modification of the following pharmacological treatments: cholinesterase inhibitor, memantine, antidepressant, or antipsychotic drugs. Low-dose benzodiazepines for insomnia were allowed during the study.

Each patient had a caregiver who supervised treatment compliance.

Demographic, neuropsychological, and anatomical details are shown in Table 1.

According to a previous multicenter controlled study, ${ }^{25}$ sample size calculation was performed considering an effect size of 1.7 and a standard deviation of 2.8 of primary outcomes differences between groups. Under the assumption of normal distribution of the scores and considering an $\alpha$ level of .05 , a sample size of 30 subjects resulted in a power greater than $70 \%$ and was thus believed to be adequate for this trial.

Moreover, 22 healthy controls (HC) who were agematched and sex-matched to the PWA (73.2 \pm 5.1 years; 8 males/14 females; MMSE score $>28$ ) were also included in the fMRI analysis for the description of the typical activation pattern and for the definition of regions of interest to be used for the analyses on PWA data.

The study was approved by the Ethics Committee of Don Gnocchi Foundation, and informed written consent was obtained from all the included subjects and their caregivers (or a legally acceptable representative if applicable and if different from the caregiver) before study initiation.

The trial was registered at www.clinicaltrials.gov (NCT01893398).

\section{Randomization and Masking}

The target population was stratified by gender and randomly assigned (1:1 ratio) to MST (tMST) or a usual care program (no stimulation treatment, ntMST) in a single-blind 
Table I. Baseline Demographics, Neuropsychological, and Anatomical Characteristics.

\begin{tabular}{lccc}
\hline & tMST Participants & ntMST Participants & Group Comparison $^{\mathrm{a}}$ \\
\hline Demographic characteristics & & & \\
Age, years (mean \pm SD) & $75.61 \pm 5.86$ & $76.50 \pm 5.65$ & $\mathrm{~ns}$ \\
Gender (male-female) & $13: 15$ & $10: 14$ & $\mathrm{~ns}$ \\
Level of education, years (mean \pm SD) & $8.61 \pm 3.75$ & $9.43 \pm 4.42$ & $n s$ \\
CDR, median [range] & $1.5[1-2]$ & $1.5[1-2]$ & $n s$ \\
MMSE (mean \pm SD) & $21.54 \pm 3.78$ & $22.04 \pm 2.61$ & $n s$ \\
NPI & $15.70 \pm 11.21$ & $14.28 \pm 8.50$ & $n s$ \\
Anatomical characteristics & & & \\
Left hippocampal volume $\left(\mathrm{mm}^{3}\right)$ & $2751 \pm 526$ & $2704 \pm 449$ & $n$ \\
Right hippocampal volume $\left(\mathrm{mm}^{3}\right)$ & $2806 \pm 580$ & $2960 \pm 558$ & $n s$ \\
\hline
\end{tabular}

Abbreviations: tMST, people treated with Multidimensional Stimulation group Therapy; ntMST, people with usual care program; CDR, Clinical Dementia Rating scale; MMSE, Mini-Mental State Examination; NPI, Neuropsychiatric Inventory scale.

${ }^{a}$ Sociodemographic and neuropsychological variables were compared at baseline using $t$ test or $\chi^{2}$ as appropriate. Data are relative to PWA who completed the trial (52 subjects).

parallel-group study. Randomization was conducted by an independent operator, who was not involved in assessment and treatment using a computer algorithm (http://graphpad. com). Participants and their caregivers were instructed not to discuss the nature of their therapy with the research assistants who did the assessments. Outcome measures were collected by researchers blinded to group allocation.

\section{Intervention}

The MST program involved 3 levels of treatment. The first level was focused on PWA, the second level involved the caregiver, whereas the third one included the dyad PWA-caregiver.

Level I-PWA. The PWA performed 30 rehabilitation sessions (2.5 hours a day, 3 days a week) in a room with a kitchen area, table and chairs, and materials necessary to carry out recreational-occupational activities. MST was administered by a psychologist and a rehabilitation therapist, both specialized in cognitive rehabilitation. Tight interaction between participants and therapists was an essential feature of our program: as previously noted, ${ }^{30}$ an attractive environment or the mere presence of the staff is insufficient to evoke in PWA the exercise of their full functional capacities. The treatment involved 4 steps: (a) Reality Orientation activities and cognitive exercises (about 45-minute); (b) physical activity (about 30-minute); (c) occupational activities of daily living (about 30-minute); (d) recreational activities (about 45- minute).

Level 2-Caregiver. All caregivers of PWA had a single support interview with a psychologist at the beginning and at the end of the training. In these moments, family caregivers could freely express their psychological sufferance and their practical difficulties. Caregivers also followed a standardized short group educational program with a rehabilitation therapist. The program touched on several points: AD clinical picture, pathogenetic mechanism, pharmacological therapy and recent advances in research, coping with behavioral problems, as well as legal and social aspects. The second level was offered $(a)$ to collect data about past preferences and personality of the PWA in order to integrate this information into the rehabilitation program; (b) to offer psychological support to the caregiver; and (c) to promote the detection of practical coping solutions. Moreover, during psychoeducational meetings, caregivers were trained by the therapist in order to continue the treatment at home (see Level 3).

Level 3-Dyad PWA and Caregiver. All subjects performed further stimulation at home: aerobic physical activity and specific but simple cognitive activities every day. Level 3 was introduced to improve in the amount and intensity of the MST treatment and to favor a positive PWA-caregiver interaction at home (eg, strategies of practical coping solutions).

\section{Procedures}

As shown in timeline (Figure 1), assessments were done in both groups at baseline ( $\left.\mathrm{T}_{-} 0\right)$ and after 10 weeks $\left(\mathrm{T}_{-} 1\right)$. Only in the tMST group a follow-up evaluation was collected after 22 weeks, because the ntMST group was treated with MST for ethical purposes in accordance with the recommendations of the local ethics committee.

The tools used as primary outcome measures in this trial were the following: (a) Alzheimer's Disease Assessment Scale-Cognitive subscale (ADAS- $\left.\operatorname{cog}^{31}\right)$; (b) the Functional Living Skills Assessment Scale $\left(\mathrm{FLSA}^{32}\right) ;(c)$ the Neuropsychiatric Inventory scale $\left(\mathrm{NPI}^{33}\right)$; and $(d)$ quality of life measures (Short Form 36 health survey questionnaire-SF-36 $6^{34}$ ). Changes in cognitive (ADAS-cog), 


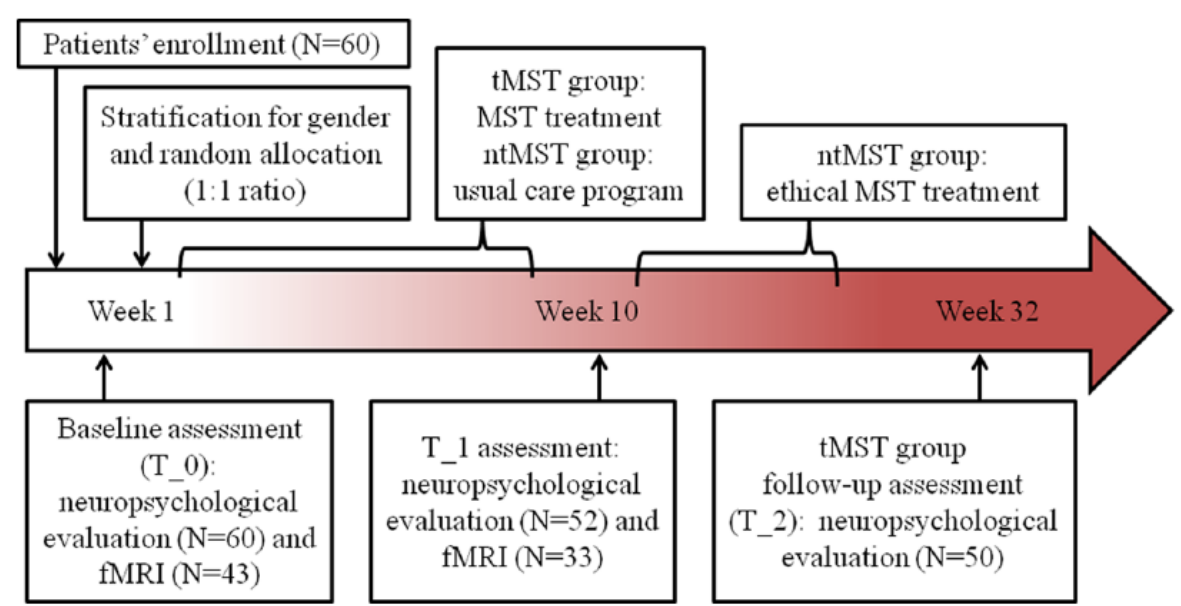

Figure I. Timeline of the trial. Timing and duration of the various procedures used in the trial.

functional (FLSA), behavioral (NPI) status, and in physical well-being (SF-36) were assessed by an experienced neuropsychologist blinded to the treatment.

Finally, we used fMRI with a language task (paced-overt verbal fluency task $\mathrm{k}^{35}$ ) to detect possible changes (T1 vs T0) in brain activation patterns in PWA as a surrogate biomarker of MST efficacy.

MRI Acquisition Protocol. MRI scans were obtained using a 1.5-Tesla scanner (Magnetom Avanto, Siemens). The protocol included the following: (a) a structural MRI study with T1, $\mathrm{T} 2$, and FLAIR weighted images to exclude subjects with pathological brain abnormality; (b) fMRI echo planar images with blood oxygenation level dependent (BOLD) contrast; and $(c)$ a morphological T1-weighted MPRAGE sequence, used as reference for fMRI analysis and for the calculation of structural measures (hippocampal volumes) with FSL (FMRIB's Software Library; www.fmrib.ox.ac.uk/fsl).

fMRI Stimuli/Design. We adopted the verbal fluency paradigm described by Basho and colleagues ${ }^{35}$ to test language function. This fMRI task was chosen because it allows an appropriate response monitoring and a tight control over and reduced individual variability of task performance, making it suitable for the application in patients with cognitive deficits. The functional sequence included 6 experimental blocks alternated with 6 control blocks ( 30 seconds for block) for a total duration of 6 minutes (see Supplemental Methods for details, available online at http://nnr.sagepub.com/content/by/supplemental-data).

Before the fMRI session, all participants completed a practice session, which included different stimuli from those presented in the scanner. For stimuli presentation and overt responses collection, we used an MRI-compatible visual and sound system (VisuaStim Digital system from
Resonance Technology Inc), while the use of E-Prime software 2.0 (http://www.pstnet.com) ensured exact timing of prompts.

\section{Statistical Analysis}

Statistical analysis on primary outcome measures was performed using SAS software package 9.2. Sociodemographic, clinical, and neuropsychological variables were compared at baseline using $t$ test or $\chi^{2}$ test as appropriate.

The primary outcome measures in this study were changes in neuropsychological test and scale scores from baseline. We modeled these changes at 10 weeks. Only in the tMST group there were changes also evaluated at 22 weeks. To account for variable skewness, nonparametric analysis and variables normalization using Blom's transformation were performed. Mann-Whitney nonparametric comparison of score differences was performed to identify statistically significant results. When variables resulted in a statistically significant difference, the analysis was confirmed using the analysis of covariance of normalized scores. Score differences were described using mean and standard deviation of retro-transformed Blom's variables. Categorical variables were described by percentages. The least square differences and $R^{2}$ model fitting were reported in Table 2 . An $\alpha$ value of .05 was considered statistically significant, and all comparisons were 2-tailed. Finally, score modifications at 22 weeks in the tMST group were evaluated by means of regression analysis over time considering previous values at baseline and 10 weeks.

\section{fMRI Data Analysis}

To investigate for fMRI task performance differences between groups at baseline and at 10 weeks, a logistic 
Table 2. Analysis of Covariance Results for All Test/Scales and Statistically Significant Subscales of ADAS-Cog.

\begin{tabular}{|c|c|c|c|}
\hline Test and Subscales & tMST & ntMST & $P$ Value \\
\hline \multicolumn{4}{|c|}{ Alzheimer's Disease Assessment Scale } \\
\hline Global score $^{\mathrm{a}}$ & $22.3(0.66)$ & $23.6(0.83)$ & .344 \\
\hline Word recall & $1.16(0.10)$ & $1.41(0.11)$ & .045 \\
\hline $\begin{array}{l}\text { Naming objects and } \\
\text { fingers }\end{array}$ & $\mathrm{I} .07(0.10)$ & $1.48(0.11)$ & .004 \\
\hline $\begin{array}{l}\text { Remembering test } \\
\text { instruction }\end{array}$ & $1.13(0.10)$ & $1.45(0.11)$ & .061 \\
\hline Spoken language ${ }^{\mathrm{b}}$ & $0.89(0.13)$ & $1.27(0.14)$ & .010 \\
\hline \multicolumn{4}{|c|}{ Functional Living Skills Assessment } \\
\hline Global score $\mathrm{a}^{\mathrm{a}}$ & $99.6(1.52)$ & $98.5(1.69)$ & .649 \\
\hline \multicolumn{4}{|c|}{ Neuropsychiatric Inventory Scale } \\
\hline Global score ${ }^{a}$ & $13.2(1.14)$ & I7.5 (I.35) & .019 \\
\hline Distress global score & $6.9(0.55)$ & $8.6(0.65)$ & .054 \\
\hline \multicolumn{4}{|c|}{ Short Form-36 health survey questionnaire } \\
\hline $\begin{array}{l}\text { Mental component } \\
\text { scale }\end{array}$ & $43.4(1.37)$ & 43.9 ( 1.59$)$ & .830 \\
\hline $\begin{array}{l}\text { Physical component } \\
\text { scale }\end{array}$ & $47.6(1.23)$ & $47.5(1.42)$ & .992 \\
\hline
\end{tabular}

Abbreviations: ADAS-Cog, Alzheimer's Disease Assessment Scale; tMST, people treated with Multidimensional Stimulation group therapy; ntMST, people with usual care program.

Statistically significant $P$ Values $(P<0.05)$ were reported in italics. ${ }^{\text {aModel }} R^{2}>0.60$.

${ }^{\mathrm{b}}$ Mann-Whitney U-test $P<.05$.

analysis was performed where percentages of correct task was the response variable and group by time interaction an explanatory covariate. To account for over dispersion of the response, the Williams scaling criterion was applied.

Statistical analysis on fMRI data was performed using SPM8 (http://www.fil.ion.ucl.ac.uk/spm). Images were realigned, coregistered, normalized (MNI space), and spatially smoothed. Single subject statistical analysis (first level) was then performed with a general linear model (GLM) to detect the activation areas during the task (t-contrast: categorical-fluency vs control-condition). The corresponding contrast images (1 image for each subject, obtained as the product between the GLM parameter estimates-betas-and the contrast vector) entered in the group analyses (second level).

First, to describe the main effect of the language task (typical activation pattern) and to extract regions of interest (ROIs) running Marsbar (http://marsbar.source-forge.net/) to be used as a priori ROIs for subsequent analyses on PWA (see below), we performed a 1-sample $t$ test in the HC group (supplemental Figure S1 and Table S1).

The following second-level analyses were then performed in PWA: (a) 1-sample $t$ tests in the PWA (both tMST and ntMST) at T 0 to describe the main effect of the language task at baseline; (b) 1 -sample $t$ tests in the PWA (both
tMST and ntMST) at T_1 to describe the main effect of the language task after 10 weeks; $(c)$ a flexible-factorial analysis including 3 factors (subject definition; group [tMST or ntMST]; time [T_0 or T_1]) to test the effect of treatment (tMST vs ntMST, group-by-time interaction: contrast 1 : tMST [T_1 > T_0] > ntMST [T_1 > T_0]; contrast 2: ntMST [T_1 > T_0] $>$ tMST [T_1 > T_0]). Normalized grey matter volume was included as a covariate. The maps resulting from the second-level analyses on PWA were thresholded with 2 approaches: first, we performed a statistical analysis within the ROIs identified in the control group by using correction for multiple comparisons (family-wise error [FWE], $P<.05)$; then, for exploratory purposes, nonhypothesized group differences outside the ROIs were described considering an uncorrected $P<.001$ threshold with 10 or more contiguous voxels.

Finally, to investigate the relationship between changes in behavior and changes in magnitude of activation in tMST compared to ntMST, we performed an additional regression analysis considering score changes by groups. For each PWA we generated a single contrast map $\left(T_{-} 1>T_{-} 0\right)$ and we extracted the mean beta value within the 10 ROIs identified as critical for language functioning in the control group (see Table S2) running Marsbar tool (http://marsbar.sourceforge.net/). Then, we plotted ADASCog delta score (T_1 minus T_0 scores) against the beta values for all PWA subjects by groups in a linear regression analysis.

\section{Results}

The statistical analyses consisted of 28 PWA given MST and 24 given standard treatment of care (Figure 2). The 2 groups were similar at baseline in sociodemographic characteristics (age $P=.58$; gender $P=.95$; education level $P=$ .47 ), global cognitive level (MMSE, $P=.58$ ), and brain hippocampal volumes (left hippocampus $P=.76$; right hippocampus $P=.38$; Table 1 )

\section{Neuropsychological and Behavioral Results}

The results obtained by PWA and the comparison between the 2 groups (tMST vs ntMST) on test and scales are summarized in Table 2. Behavioral symptoms showed a significant reduction in tMST with respect to ntMST (NPI global score $P=.019$ ). The tMST group also obtained a significant improvement in language (spoken language $P=.01$; naming objects and fingers $P=.004$ ) and memory (word recall $P=.045$; remembering test instructions $P=.041)$ subscales of ADAS-Cog. No statistically significant differences between baseline and $\mathrm{T} 1$ were observed in the measures of functional status (FLSA $P=.649$ ) and physical well-being (SF-36 MCS $P=.83$; SF-36 PCS $P=.99$ ).

Finally, we obtained no statistically significant slope of the regression model for any of the previous described 


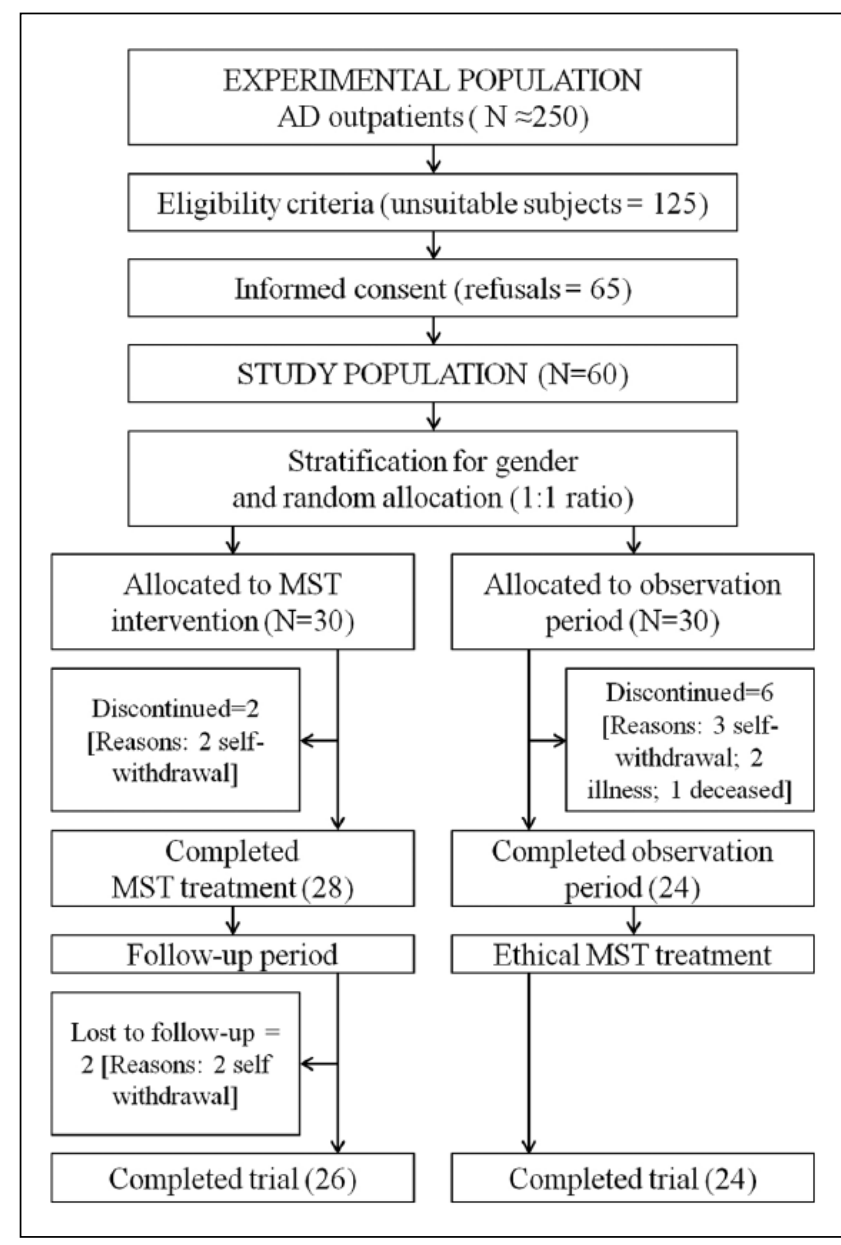

Figure 2. Trial profile.

cognitive (language and memory subscale of ADAS-Cog) and behavioral (NPI values) measures considered in the tMST group at 22 weeks with respect to baseline and 10 weeks results.

\section{fMRI Results}

Regarding fMRI, only data relative to 22 PWA (12 tMST and $10 \mathrm{ntMST}$ ) entered the second-level fMRI analyses (the details of this subsample are shown in Supplemental results and Table S2). The task performance of the included subjects on correct responses was $83.1 \%$ to $84.2 \%$ (tMSTntMST) at baseline and $88.9 \%$ to $87.8 \%$ (tMST-ntMST) after 10 weeks. The fMRI task performance differences within group (tMST $P=.37$; ntMST $P=.70$ ) and between groups at baseline and 10 weeks were not statistically significant $(P=.47)$.

In line with previous findings, ${ }^{35,36}$ the healthy controls exhibited a typical activation pattern of paced overt verbal fluency task: areas related to category-driven word generation (left inferior and middle frontal cortex, the superior and middle temporal regions, left thalamus and lentiform nucleus), areas associated with paced response and overt articulation (cingulate cortex, right superior parietal cortex, insular cortex, thalamus, lentiform nucleus and cerebellum; see supplemental Figure S1 and Table S1 for details). These clusters were chosen as a priori ROIs for subsequent analyses.

As shown in Figure 3A, a significant activation $\left(P_{\mathrm{FWE}}<\right.$ .05 ) in PWA was found at T_0 only in the left superior temporal gyrus (BA 22). For exploratory purposes, considering an uncorrected threshold $P<.001$ with 10 or more contiguous voxels, we found brain activations also in the left inferior frontal (BA 44) gyrus.

At $T$ _1 (Figure 3B), the activation pattern found at baseline was strongly increased, resulting in significant activations $\left(P_{\mathrm{FWE}}<.05\right)$ in the left inferior frontal and superior temporal gyri, the right superior temporal cortex (BA 22-41), the left cingulate cortex (BA32), the bilateral basal ganglia, thalamus, and the right superior parietal lobule (BA 7). Considering an uncorrected threshold $P<.001$ with more than 10 contiguous voxels, we found an additional brain activation in the right cerebellum.

Results of the flexible factorial analysis evaluating the effect of treatment (group-by-time interaction) are reported in Table 3: the tMST group showed a significant intervention-related increase in activation (contrast 1: tMST [T_1 $>$ T_0] > ntMST [T_1 > T_0], $\left.P_{\text {FWE }}<.05\right)$ of the bilateral superior temporal gyrus (right $>$ left) and the right lentiform nucleus and thalamus. Considering an uncorrected threshold $P<.001$ with more than 10 contiguous voxels, we observed in the tMST group an additional increase in brain activation in the right insular cortex with respect to the ntMST due to the MST intervention. No significant activation were found with the opposite contrast (contrast 2: ntMST [T_1 > T_0] > tMST [T_1 > T_0]). The plots in Figure 4 illustrate the mean beta values for each group (ntMST and tMST) at T_0 and T_1 extracted from the 4 clusters described in Table 3.

Finally, the results of regression analysis are reported in Figure 5. In the tMST group we found a significant correlation between increase in magnitude of activation in the left superior temporal gyrus (BA 22/41-ROI 2), precuneus (BA7-ROI 5), left thalamus (ROI 9), and change in ADAS-Cog. Conversely, only one significant correlation was found in ntMST group between the left superior temporal gyrus (ROI 2) and change in ADAS-Cog. The negative correlation is due to the ADAS-Cog score: a decrease in ADAS-Cog score is indicative of cognitive improvement.

\section{Discussion}

The results obtained in this RCT study supported the initial hypothesis that MST has an impact on at least $2 \mathrm{AD}$ domains: behavior-reduction of BPSD and improvement in some cognitive abilities. 

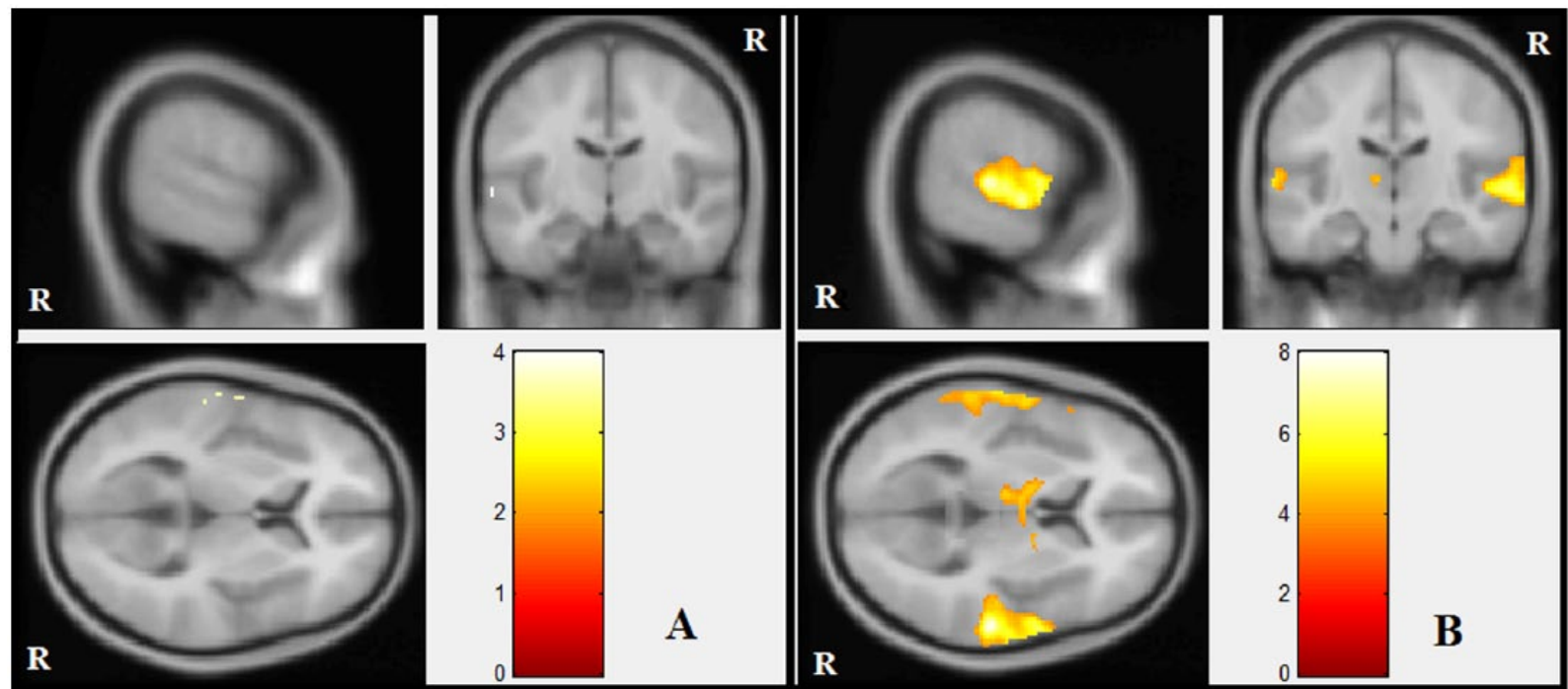

A
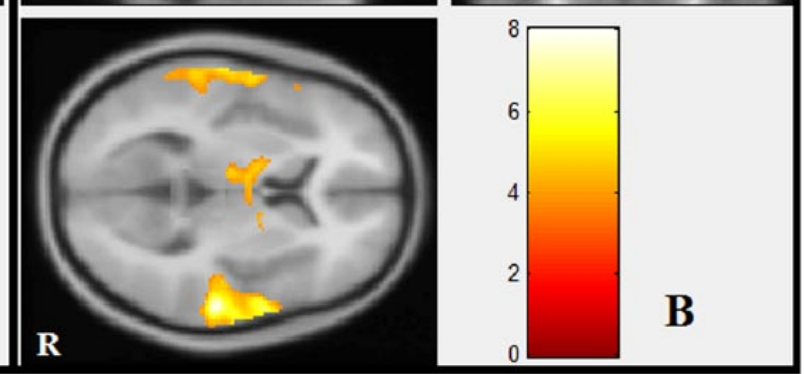

Figure 3. Main effect of paced-overt verbal fluency task in PWA: brain areas showing a significant activation at baseline (A) and after 10 weeks (B).

Data are relative to PWA (both tMST and ntMST) who completed the $2 \mathrm{fMRI}$ assessments $(\mathrm{N}=22 ; \mathrm{P}$ Abbreviations: PWA, people with Alzheimer's disease; tMST, people treated with multidimensional stimulation group therapy; ntMST, people with usual care program; $R$, right hemisphere.

Table 3. Maxima of Regions Showing Significantly Higher Brain Activation From T_0 to T_I in tMST Group With Respect to Changes Observed in ntMST Group ${ }^{\mathrm{a}, \mathrm{b}}$.

\begin{tabular}{lcccccccc}
\hline \multicolumn{10}{c}{ Brain Activations } \\
\hline$x$ & $y$ & $z$ & $\begin{array}{c}\text { Cluster } \\
\text { Size }\end{array}$ & Side & Brain Area & BA & z-Value \\
\hline-50 & -32 & 8 & 18 & L & Sup temporal Gy & $4 I \_22$ & 3.48 & $*$ \\
58 & -10 & 8 & 81 & $\mathrm{R}$ & Sup temporal Gy & $4 I \_22$ & 3.57 & $*$ \\
44 & -32 & 18 & $\mathrm{I}$ & $\mathrm{R}$ & Insula & 13 & 3.57 & \\
16 & -8 & 0 & 42 & $\mathrm{R}$ & Thalamus & - & 4.28 & $*$ \\
\hline
\end{tabular}

Abbreviations: Sup, superior; Inf, inferior; R, right; L, left; Gy, gyrus; BA, Brodmann area; $\mathrm{tMST}$, people treated with multidimensional stimulation group therapy; ntMST, people with usual care program; fMRI, functional magnetic resonance imaging; ROI, region of interest.

${ }^{a}$ Flexible factorial analysis (group per factor interaction). Data are relative to PWA (both tMST and ntMST) who completed the 2 fMRI assessments $(N=22)$.

${ }^{b}$ Results are relative to $P<.001$ uncorrected.

*Clusters FWE corrected for multiple comparisons within the ROls extracted from the control group.

Regarding the behavioral domain, these results confirm our previous findings $\mathrm{s}^{24,25}$ and are in line with other nonpharmacological studies that reported a reduction of BPSD with the single components of our therapy. ${ }^{37-41}$ MST can be easily implemented with both ambulatory patients, such as in our case, and institutionalized ones and applies to people with BPSD in the moderate range. As severe psychotic symptoms (hallucinations, significant delusions, or evident aggression) would preclude the participation in group therapy, we did not include this type of PWA in this study. Examining the single items of NPI, we observed that the BPSD which benefit most from MST were depression, anxiety, irritability, and aberrant motor behavior. Teri and her group ${ }^{37}$ observed a decrease of depression in PWA after a treatment based on physical activity. Interestingly, different mechanisms were recently suggested to support the notion that exercise may have the potential to slow the decline of AD ${ }^{19,42}$ There is the possibility that new brain cells can be created within the critical parts of the hippocampus in PWA who are physically fit. There is also the possibility that increased neural connectivity can be obtained through the neuroplastical activity of BDNF, whose levels appear to be increased by exercise. ${ }^{43}$ Gitlin et al have reported ${ }^{38,39}$ a reduction of BPSD as a result of an occupational therapy intervention with empowerment of caregiver coping abilities. They also showed that nonpharmacologic management of BPSD is a recommended, cost-effective treatment as it can significantly improve quality of life and patient-caregiver satisfaction. ${ }^{15}$ Reduction of apathy, a BPDS symptom very difficult to treat, has been reported with an occupational therapy approach, ${ }^{40}$ and even more "cognitive-oriented" techniques. ${ }^{44}$ However, we believe that MST, a multidimensional treatment including all of these techniques, is the most suitable to obtain positive results, in concordance with Arkin's opinion: "Clinically, there is much to be said for leaving the combined intervention intact. By offering a 


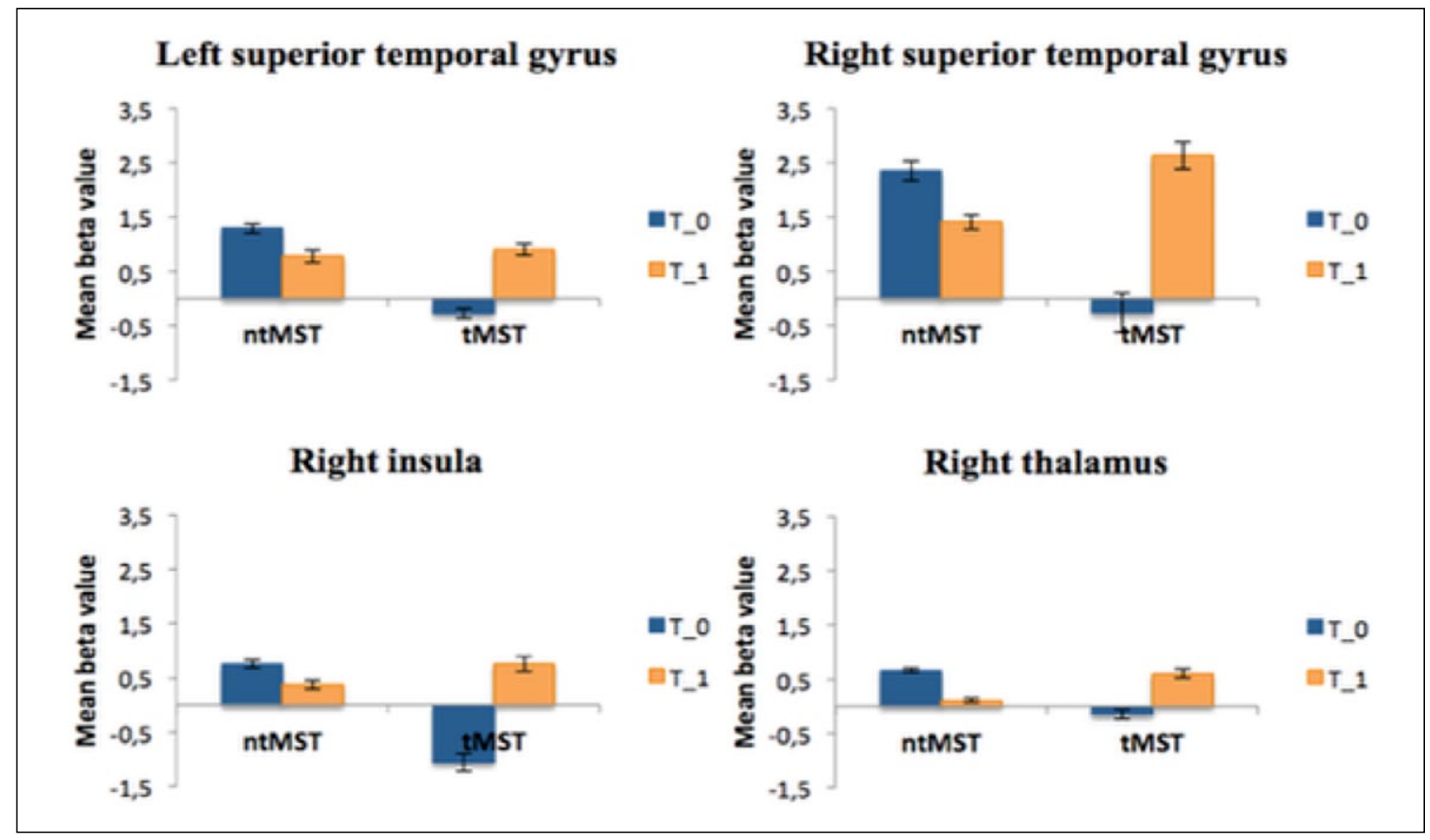

Figure 4. Plots show mean beta values for each group (ntMST and tMST) at baseline (T_0) and after 10 weeks (T_l) extracted from the 4 clusters refer to contrast: tMST $\left(T_{-} I>T_{-} 0\right)>$ ntMST $\left(T_{-} I>T_{-} 0\right)$ (see Table 3).

Error bars indicate the standard error of the mean.

Abbreviations: tMST, people treated with multidimensional stimulation group therapy; ntMST, people with usual care program.

variety of activities, you are providing multiple and different opportunities for participants to be successful." 21 Moreover, we are now well aware of the possible side effects and risks of psychotropic medications in PWA. Consequently, nonpharmacological interventions have become the first-line approach for BPSD. ${ }^{15,41}$ In this perspective, the MST approach can be helpful to avoid the excessive use of these drugs for BPSD.

As far as the cognitive domain is concerned, we found an improvement of language and memory ADAS-Cog subscales. This is in line with a recent study by Hall and colleagues ${ }^{13}$ investigating the effects of $\mathrm{CST}^{9}$ on specific areas of cognition. They attribute the changes in language and memory to the language-based nature of CST that enhances neural pathways responsible for processing of syntax, possibly aiding also verbal recall. Moreover, they hypothesize that these CST-induced changes promote the functioning of alternative neural pathways. ${ }^{45}$ As with $\mathrm{CST},{ }^{12}$ our MST was group-based and included, in the first step of level 1 (Reality Orientation activities and cognitive exercises), many tasks aimed to reinforce oral and written language. Furthermore, group therapy has been shown to facilitate social interaction among participants and to consequently promote language function, ${ }^{46}$ thereby reducing the additional handicap due to social isolation of PWA. ${ }^{17}$ For the first time, we are able to discuss also the neural mechanisms that may underlie these intervention-related changes on language by using fMRI. The fMRI results at T_1 with respect to T_0 showed increased brain activation in left frontotemporal areas (BA 22/44), right superior temporal cortex, bilateral basal ganglia, cingulate cortex, and right superior parietal cortex. In the tMST group, an intervention-related increase in activations was found in the bilateral superior temporal cortex (BA 22/41), the thalamus, and the anterior insular cortex. It is known that thalamus and basal ganglia play an important role in word generation, and recent data suggest that the thalamus acts as a central monitor for language-specific cortical activities, supported by the basal ganglia in both perceptual and productive language execution. ${ }^{47}$ Moreover, the insular cortex plays an important role in speech and emotional experience, and it has been demonstrated that hypometabolism of the anterior insula is associated with progressive nonfluent aphasia. ${ }^{48}$ Furthermore, MRI studies have shown that the insula is interconnected with the temporal and orbitofrontal cortices and inferior frontal gyrus. ${ }^{49}$ The obtained fMRI intervention-related changes may reflect a restoration of neural function in the underactive language 


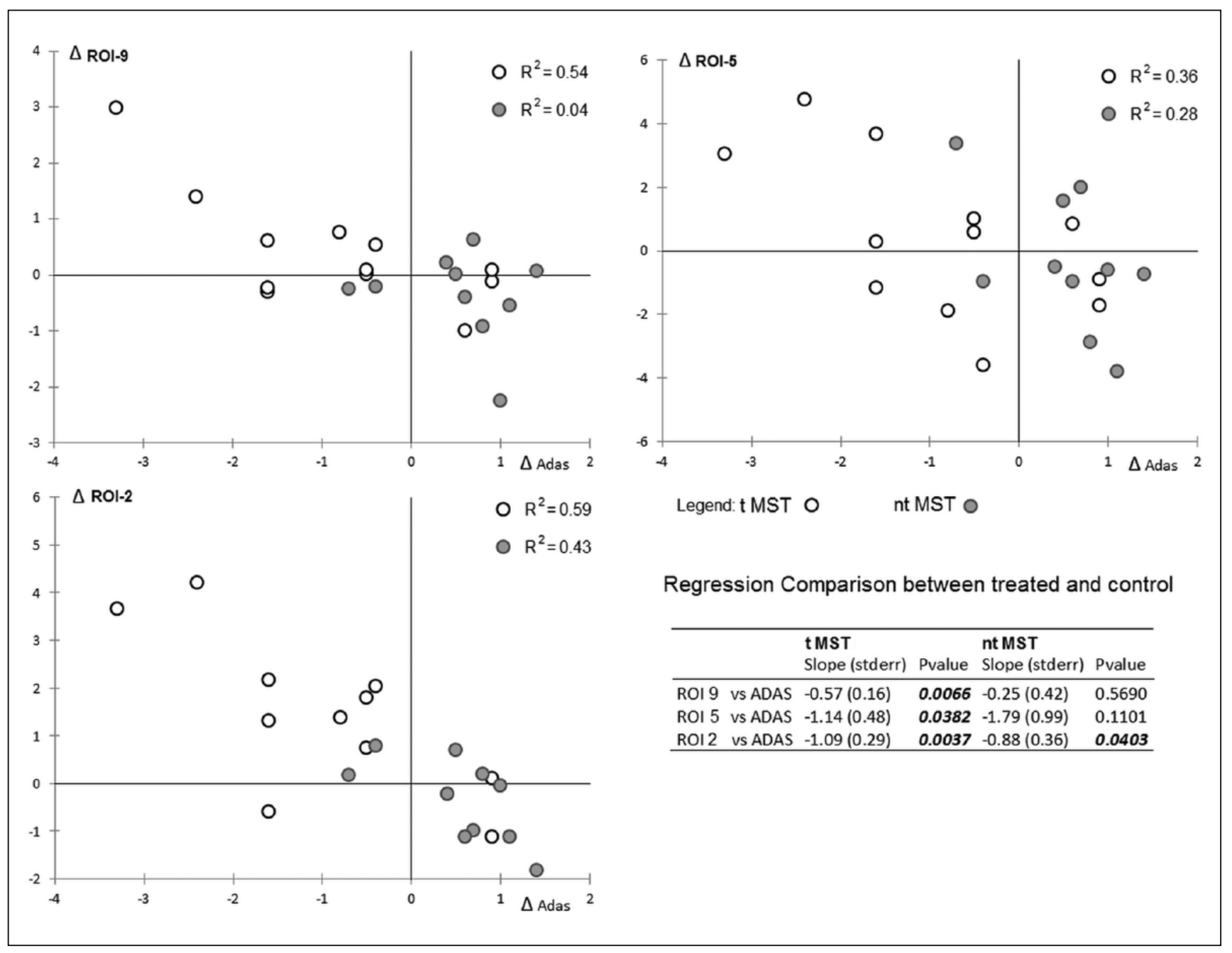

Figure 5. Scatterplots show statistically significant results of regression analysis considering score changes by groups.

We plot ADAS-Cog delta score (T_I minus T_0) against the betas values ( $\left.T_{-} I>T_{-} 0\right)$ for all PWA subjects by groups (tMST, ntMST).

Abbreviations: tMST, people treated with multidimensional stimulation group therapy; ntMST, people with usual care program; ROI region of interest.

network via the compensatory strengthening of specific brain regions. ${ }^{45}$ Further intervention-related changes, although using an encoding-recognition task, were described using fMRI by Clare and colleagues. ${ }^{6}$ They performed an RCT on cognitive rehabilitation in people with dementia and a subset of participants also underwent fMRI scanning. Four right brain regions forming part of the network for visual associative learning (fusiform face area, medial prefrontal gyrus, parahippocampal cortex, and temporal parietal junction) showed increased activation due to the treatment and the authors inferred that these results primarily reflect restoration of function in PWA.

The changes we saw in fMRI support the notion that even the AD brain still has plasticity resources and can react to positive environmental stimuli. Obviously, intervention in the predementia stage could improve the memory domain even more so, assuming that the more precocious the cognitive intervention is, the better the result will be. In this perspective, it is interesting to note that some recent studies reported increased hippocampal activity after memory training in MCI people, suggesting that the hippocampus may retain sufficient neuroplasticity in this clinical situation. $^{50,51}$ However, even if this will probably be the right strategy in the future, our study, along with others, ${ }^{13,22,39}$ demonstrates that cognitive stimulation can be useful in people with overt AD.

Regarding the long-lasting effect of the treatment, our data showed that the improvement in cognitive and behavioral areas is preserved at 22 weeks. The persistence of effects, along with generalization of gain in everyday life, is the critical point of nonpharmacological therapies and is being explored in ongoing trials. ${ }^{11}$ The necessity of a longterm treatment to maintain positive effects engenders the problem of the treatment costs. However, it must 
be considered that MST is a group treatment, potentially allowing for a more effective use of personnel resources when compared to individual-specific techniques. Moreover, relatives and caregivers assisting PWA at home can be trained for this type of treatment to reinforce and make the benefits more enduring. ${ }^{24}$

We consider the differences in cognition and behavior are clinically meaningful. PWA in the treated group showed a reduction of BPSD of almost $20 \%$ from the baseline level. Moreover, this result was due above all to reduction of symptoms such as depression, irritability, and aberrant motor behavior, which have a particular negative impact on caregivers in our clinical experience. ${ }^{52}$ We consider this result clinically significant, because it can allow a reduction of the use of antipsychotic drugs, whose harmful effects on PWA is now well known. As for the clinical value of results in the cognitive domain, we believe that obtaining even a moderate improvement of language and memory is a meaningful result: memory impairment is the principal symptom of PWA and is a source of depression for them and of burden for caregivers (eg, due to repetitive questioning $)^{53}$; impairment of language, on the other hand, favors social isolation and has particular negative sequelae for the interaction with family members, increasing their burden of care. In the progression from moderate to severe stages in $\mathrm{AD}$, worsening language abilities, or aphasia, has been suggested to have more clinical relevance than other domains. As a matter of fact, decline in language has been shown to correlate with noncognitive items, such as personal care, hobbies, occupations, and behavior. ${ }^{53}$

Overall, despite the significant results in improving different aspects of PWA, our study is not without limitations. Although the improvement in cognitive and behavioral areas was preserved in the tMST group at 22 weeks, the length of follow-up was relatively short. Thus, whether or not the treatment effect produced by the MST approach is long-lasting remains unknown at this time. Future studies should investigate MST's efficacy over longer durations of time. Furthermore, a direct comparison between the tMST and ntMST groups at T 2 was not possible due to ethical concerns. Our ethical committee requested that we also treat the ntMST group after $\mathrm{T} 1$ evaluation.

In conclusion, MST is a nonpharmacological approach for mild to moderate PWA that was shown to have a positive impact on behavioral and cognitive functions, enhancing patients' motivation, promoting the use of their remaining function, and preventing further loss in agreement with the statement "use it or lose it."

\section{Declaration of Conflicting Interests}

The author(s) declared no potential conflicts of interest with respect to the research, authorship, and/or publication of this article.

\section{Funding}

The author(s) disclosed receipt of the following financial support for the research, authorship, and/or publication of this article: This work was supported by 2011-2012 Ricerca Corrente (Italian Ministry of Health).

\section{References}

1. McKhann GM, Knopman DS, Chertkow H, et al. The diagnosis of dementia due to Alzheimer's disease: recommendations from the National Institute on Aging-Alzheimer's Association workgroups on diagnostic guidelines for Alzheimer's disease. Alzheimers Dement. 2011;7:263-269.

2. Farina E. The language revolution-editorial. Nonpharmacol Ther Dement. 2012;2(2):77-80.

3. Moniz-Cook E, Vernooij-Dassen M, Woods B, Orrell M. Psychosocial interventions in dementia care research: the INTERDEM manifesto. Aging Ment Health. 2011;15: 283-290.

4. Cramer SC, Sur M, Dobkin BH, et al. Harnessing neuroplasticity for clinical applications. Brain. 2011;134(pt 6): 1591-1609.

5. Hampstead BM, Stringer AY, Stilla RF, et al. Activation and effective connectivity changes following explicit-memory training for face-name pairs in patients with mild cognitive impairment: a pilot study. Neurorehabil Neural Repair. 2011;25:210-222

6. Clare L, Linden DE, Woods RT, et al. Goal-oriented cognitive rehabilitation for people with early-stage Alzheimer disease: a single-blind randomized controlled trial of clinical efficacy. Am J Geriatr Psychiatry. 2010;18:928-939.

7. Aguirre E, Hoare Z, Streater A, et al. Cognitive stimulation therapy (CST) for people with dementia-who benefits most? Int J Geriatr Psychiatry. 2013;28:284-290.

8. Spector A, Davies S, Woods B, Orrell M. Reality orientation for dementia: a systematic review of the evidence of effectiveness from randomized controlled trials. Gerontologist. 2000;40:206-212.

9. Spector A, Thorgrimsen L, Woods B, et al. Efficacy of an evidence-based cognitive stimulation therapy programme for people with dementia: randomised controlled trial. $\mathrm{Br} J$ Psychiatry. 2003;183:248-254.

10. National Institute for Health and Clinical Excellence and the Social Care Institute for Excellence (NICE-SCIE). $A$ NICESCIE Guideline on Supporting People With Dementia and Their Carers in Health and Social Care (National Clinical Practice Guideline No. 42). London, England: British Psychological Society \& Royal College of Psychiatrists; 2007.

11. Streater A, Spector A, Aguirre E, et al. Maintenance cognitive stimulation therapy (CST) in practice: study protocol for a randomized controlled trial. Trials. 2012;13:91.

12. Spector A, Orrell M, Woods B. Cognitive stimulation therapy (CST): effects on different areas of cognitive function for people with dementia. Int J Geriatr Psychiatry. 2010;25: 1253-1258.

13. Hall L, Orrell M, Stott J, Spector A. Cognitive stimulation therapy (CST): neuropsychological mechanisms of change. Int Psychogeriatr. 2013;25:479-489. 
14. Woods B, Aguirre E, Spector AE, Orrell M. Cognitive stimulation to improve cognitive functioning in people with dementia. Cochrane Database Syst Rev. 2012;(2):CD005562.

15. Gitlin LN, Hodgson N, Jutkowitz E, Pizzi L. The cost-effectiveness of a nonpharmacologic intervention for individuals with dementia and family caregivers: the tailored activity program. Am J Geriatr Psychiatry. 2010;18:510-519.

16. Kolanowski A, Litaker M, Buettner L, Moeller J, Costa PT Jr. A randomized clinical trial of theory-based activities for the behavioral symptoms of dementia in nursing home residents. $J$ Am Geriatr Soc. 2011;59:1032-1041.

17. Teri L, Logsdon RG, McCurry SM. Exercise interventions for dementia and cognitive impairment: the Seattle protocols. $J$ Nutr Health Aging. 2008;12:391-394.

18. Thune-Boyle IC, Iliffe S, Cerga-Pashoja A, Lowery D, Warner J. The effect of exercise on behavioral and psychological symptoms of dementia: towards a research agenda. Int Psychogeriatr. 2012;24:1046-1057.

19. Erickson KI, Voss MW, Prakash RS, et al. Exercise training increases size of hippocampus and improves memory. Proc Natl Acad Sci U S A. 2011;108:3017-3022.

20. Probst M, Knapen J, Poot G, Vancampfort D. Psychomotor therapy and psychiatry: what's in a name? Open Complement Med J. 2010;2:105-113.

21. Arkin S. Language-enriched exercise plus socialization slows cognitive decline in Alzheimer's disease. Am J Alzheimers Dis Other Demen. 2007;22:62-77.

22. Olazaran J, Reisberg B, Clare L, et al. Nonpharmacological therapies in Alzheimer's disease: a systematic review of efficacy. Dement Geriatr Cogn Disord. 2010;30:161-178.

23. Farina E, Fioravanti R, Chiavari L, et al. Comparing two programs of cognitive training in Alzheimer's disease: a pilot study. Acta Neurol Scand. 2002;105:365-371.

24. Farina E, Mantovani F, Fioravanti R, et al. Evaluating two group programmes of cognitive training in mild-to-moderate $\mathrm{AD}$ : is there any difference between a "global" stimulation and a "cognitive-specific" one? Aging Ment Health. 2006;10:211-218.

25. Farina E, Mantovani F, Fioravanti R, et al. Efficacy of recreational and occupational activities associated to psychologic support in mild to moderate Alzheimer disease: a multicenter controlled study. Alzheimer Dis Assoc Disord. 2006;20:275-282.

26. Magni E, Binetti G, Bianchetti A, Rozzini R, Trabucchi M. Mini-Mental State Examination: a normative study in Italian elderly population. Eur J Neurol. 1996;3:198-202.

27. Hughes CP, Berg L, Danziger WL, Coben LA, Martin RL. A new clinical scale for the staging of dementia. Br J Psychiatry. 1982;140:566-572.

28. Oldfield RC. The assessment and analysis of handedness: the Edinburgh Inventory. Neuropsychologia. 1971;9:97-113.

29. Spinnler H, Tognoni P. Standardizzazione e taratura italiana di test neuropsicologici. Ital J Neurol Sci. 1987;86:1-20.

30. Wood W, Harris S, Snider M, Patchel SA. Activity situations on an Alzheimer's disease special care unit and resident environmental interactions, time use, and affect. Am J Alzheimers Dis Other Demen. 2005;20:105-118.

31. Rosen WG, Mohs RC, Davis KL. A new rating scale for Alzheimer's disease. Am J Psychiatry. 1984;141:1356-1364.
32. Farina E, Fioravanti R, Pignatti R, et al. Functional living skills assessment: a standardized measure of high-order activities of daily living in patients with dementia. Eur J Phys Rehabil Med. 2010;46:73-80.

33. Cummings JL, Mega M, Gray K, Rosenberg-Thompson S, Carusi DA, Gornbein J. The neuropsychiatric inventory: comprehensive assessment of psychopathology in dementia. Neurology. 1994;44:2308-2314.

34. Apolone G, Mosconi P. The Italian SF-36 health survey: translation, validation and norming. $J$ Clin Epidemiol. 1998;51:1025-1036.

35. Basho S, Palmer ED, Rubio MA, Wulfeck B, Muller RA. Effects of generation mode in fMRI adaptations of semantic fluency: paced production and overt speech. Neuropsychologia. 2007;45:1697-1706.

36. Price CJ. A review and synthesis of the first 20 years of PET and fMRI studies of heard speech, spoken language and reading. Neuroimage. 2012;62:816-847.

37. Teri L, Gibbons LE, McCurry SM, et al. Exercise plus behavioral management in patients with Alzheimer disease: a randomized controlled trial. JAMA. 2003;290:2015-2022.

38. Gitlin LN, Winter L, Burke J, Chernett N, Dennis MP, Hauck WW. Tailored activities to manage neuropsychiatric behaviors in persons with dementia and reduce caregiver burden: a randomized pilot study. Am J Geriatr Psychiatry. 2008;16:229-239.

39. Gitlin LN, Winter L, Dennis MP, Hodgson N, Hauck WW. A biobehavioral home-based intervention and the well-being of patients with dementia and their caregivers: the COPE randomized trial. JAMA. 2010;304:983-991.

40. Buettner L, Fitzsimmons S. Recreational therapy interventions: a fresh approach to treating apathy and mixed behaviors in dementia. Nonpharmacol Ther Dement. 2011;1(1):27-42.

41. Corbett A, Smith J, Creese B, Ballard C. Treatment of behavioral and psychological symptoms of Alzheimer's disease. Curr Treat Options Neurol. 2012;14:113-125.

42. Exercise and brain health. Preventing Alzheimer's and more. Mayo Clin Health Lett. 2013;31(1):4-5.

43. Erickson KI, Voss MW, Prakash RS, et al. Exercise training increases size of hippocampus and improves memory. Proc Natl Acad Sci U S A. 2011;108:3017-3022.

44. Vallar G, Cantagallo A, Cappa SF, Zoccolotti P. La riabilitazione neuropsicologica un'analisi basata sul metodo evidence-based medicine. Berlin, Germany: Springer; 2012.

45. Stern Y. What is cognitive reserve? Theory and research application of the reserve concept. J Int Neuropsychol Soc. 2002;8:448-460.

46. Spector A, Gardner C, Orrell M. The impact of cognitive stimulation therapy groups on people with dementia: views from participants, their carers and group facilitators. Aging Ment Health. 2011;15:945-949.

47. Klostermann F, Krugel LK, Ehlen F. Functional roles of the thalamus for language capacities. Front Syst Neurosci. 2013;16(7):32.

48. Jakab A, Molnar PP, Bogner P, Beres M, Berenyi EL. Connectivity-based parcellation reveals interhemispheric differences in the insula. Brain Topogr. 2012;25:264-271.

49. Nestor PJ, Graham NL, Fryer TD, Williams GB, Patterson K, Hodges JR. Progressive non-fluent aphasia is associated with 
hypometabolism centred on the left anterior insula. Brain. 2003;126(pt 11):2406-2418.

50. Hampstead BM, Stringer AY, Stilla RF, Giddens M, Sathian K. Mnemonic strategy training partially restores hippocampal activity in patients with mild cognitive impairment. Hippocampus. 2012;22:1652-1658.

51. Rosen AC, Sugiura L, Kramer JH, Whitfield-Gabrieli S, Gabrieli JD. Cognitive training changes hippocampal function in mild cognitive impairment: a pilot study. $J$ Alzheimers Dis. 2011;26(suppl 3):349-357.
52. Sink KM, Holden KF, Yaffe K. Pharmacological treatment of neuropsychiatric symptoms of dementia: a review of the evidence. JAMA. 2005;293:596-608.

53. Woodward M. Aspects of communication in Alzheimer's disease: clinical features and treatment options. Int Psychogeriatr. 2013;25:877-885.

54. Swaab DF, Dubelaar EJ, Hofman MA, Scherder EJ, van Someren EJ, Verwer RW. Brain aging and Alzheimer's disease: use it or lose it. Prog Brain Res. 2002;138: 343-373. 